\title{
Modeling and measurements of angular truncation for an aerosol albedometer
}

\section{F. Qian}

L. Ma

\section{J. E. Thompson \\ jon.thompson@ttu.edu}

Department of Chemistry \& Biochemistry, Texas Tech University, MS1061, Lubbock, TX 79409, USA

Department of Chemistry \& Biochemistry, Texas Tech University, MS1061, Lubbock, TX 79409, USA

In this work, we examine the angular truncation behavior and present correction factors for the aerosol albedometer previously developed in our laboratory. This new instrument makes simultaneous measurement of extinction and scattering coefficients ( $b_{\text {ext }}$ and $b_{\text {scat }}$ ) on dispersed aerosol samples. The aerosol extinction coefficient is measured with cavity ring-down spectroscopy (CRDS), and the scattering coefficient is determined through the integrating sphere nephelometer. However, all nephelometers are not able to collect light scattered from an aerosol sample very near the forward $\left(0^{\circ}\right)$ and reverse $\left(180^{\circ}\right)$ directions, due to the geometrical constraints. This can result in systematic underestimation of scattering coefficient known as truncation error. In order to account for this problem and describe scattering by aerosols more precisely, correction factors (C) for this angular non-ideality have been theoretically developed. Truncation angles $(\theta)$ were calculated upon consideration of the geometry of the sphere nephelometer. As truncation error largely depends on particle size and refractive index, C values were computed for a series of spherical, homogeneous aerosol particles with different known particle sizes and refractive indices by Lorenz-Mie theory. Measurements on size-selected, laboratory generated aerosols of known size and composition allowed empirical measurement of truncation correction factors to compare with the Mie model results. Results indicate the model we built overestimates the fraction of light not collected by the sphere. Empirically observed correction factors of $\leq 1.12$ for particles with size parameters $(\alpha)<6$ were determined. In addition, the effect of number of particles within the probe beam on the suitability of correction factors was also examined. Observations support the hypothesis that particles are rapidly transported / mixed through the probe beam, and measurement integration times as short as $52 \mathrm{~s}$ yield data that is indistinguishable from the probe region being homogeneously filled with aerosol, even at very low particle concentrations.

[DOI: http://dx.doi.org/10.2971/jeos.2012.12021]

Keywords: light scattering, aerosols, truncation, nephelometry

\section{INTRODUCTION}

The aerosol albedometer has recently been described in the literature [1]-[4]. The unique feature of this device is the capability of simultaneously measuring scattering $\left(b_{\text {scat }}\right)$ and extinction coefficients $\left(b_{\text {ext }}\right)$ on dispersed aerosol samples. The single-scattering albedo $(\omega)$ can thus be easily computed through the well-known equation:

$$
\omega=\frac{b_{\text {scat }}}{b_{\text {ext }}}
$$

Direct measurement of $b_{\text {scat }}, b_{\text {ext }}$ and $\omega$ is of keen scientific interest given these variables importance to direct climate forcing by atmospheric aerosols $[5,6]$. The albedometer performs cavity ring-down spectroscopy (CRDS) on an aerosol sample within an integrating sphere nephelometer. The CRDS measurement provides an accurate and sensitive method for determination of $b_{\text {ext }}$ [7]-[12]. Use of the integrating sphere for scatter measurement is known to significantly reduce truncation angle compared to commercially available nephelometers [13]. While angular truncation was briefly considered in an early publication describing the albedometer method, underestimation of the scattering measurement was not considered in much detail and the exact truncation performance of the device has not been considered rigorously. It is well-known that all nephelometers cannot collect light scattered from an aerosol sample very near the forward $\left(0^{\circ}\right)$ and reverse $\left(180^{\circ}\right)$ directions. If uncorrected, this results in a systematic underestimation of scattering coefficient $\left(b_{\text {scat }}\right)$. In order to account for truncation error and describe integrated scattering by aerosols more precisely, correction factors (C) for the angular nonideality have been developed for commercial devices [14, 15]. This manuscript pursues this goal for the sphere albedometer. Clearly, establishing correction factors is required to improve accuracy of measurement. In this work, truncation angles $(\theta)$ were calculated upon consideration of geometry of the integrating sphere nephelometer and exact position of the scattering particle within the sphere. As truncation error largely depends on particle size and refractive index, $\mathrm{C}$ values were computed for a series of spherical, homogeneous aerosol particles with different known particle sizes and refractive indices. Then, the optical parameters $\left(b_{\text {ext }}, \mathrm{b}_{\text {scat }}\right.$, and SSA) of laboratory generated aerosols of known size and composition were measured and compared to the calculations. In ad- 
dition, the number of particles within the probe laser beam volume in CRDS is often small due to the small volume of the beam (often $<0.1 \mathrm{~cm}^{3}$ ) and low particle concentrations used. This is known to cause measurement imprecision, but more importantly leads to a situation in which truncation correction factors may vary significantly as the exact arrangement of particles within the beam change. The effect of the number of particles within the probe beam on correction factors is also examined in this work. Such effects could be particularly detrimental for large diameter aerosols such as mineral dusts, which are known to have large sizes, and irregular morphologies [16, 17]. This manuscript presents key information required to improve the performance of the sphere albedometer for measuring aerosol scattering coefficients.

\section{METHODS}

\subsection{Estimation of truncation angle}

In order to understand the angular truncation error for the integrating sphere nephelometer, truncation angle must be determined first. Truncation angle $(\theta)$ signifies the angle at which light cannot be collected by the integrating sphere nephelometer. Figure 1(a) illustrates a schematic of the sphere nephelometer and a simple geometrical model of the truncation angle in the "forward" and "reverse" direction. We use quotations here since directionality is essentially arbitrary if the ping-pong model of ring-down spectroscopy is invoked in which light bounces between mirrors. The truncation angle $(\theta)$ for a particle located in the laser beam at a specific point in a single direction can be calculated by the following equation:

$$
\theta=\arctan \left(\frac{r}{d}\right)
$$

where $r$ is the radius of the axial hole in the sphere, and $d$ is the distance of the scattering particle from the hole of interest along the optical axis. Light is scattered both "forward" and "backwards" by a particle, so the truncation angle in opposite direction must also be considered. The truncation angle in the opposite direction $\left(\theta^{\prime}\right)$ can be expressed as:

$$
\theta^{\prime}=\arctan \left(\frac{r}{l-d}\right)
$$

where $l$ is the distance between the two holes in the sphere. For the integrating sphere nephelometer used in these experiments, the variables were $\mathrm{r}=0.3175 \mathrm{~cm}$, and $\mathrm{l}=48.1 \mathrm{~cm}$. From considering the measurement geometry it is immediately obvious that the truncation angle in either direction varies with the particle's location in the sphere. Figure 1(a) also illustrates the relationship between the truncation angle and location of the particle. For this figure, the truncation angle was computed according to the particle position (d) with $100 \mu \mathrm{m}$ spatial resolution. Particle position (d) is measured from one of the axial holes in the sphere. The red and blue lines represent the truncation angle for a particle located at specified distance from one of the axial holes. As observed, when a scattering particle approaches one of the axial holes, truncation angle approaches $90^{\circ}$ in the forward direction and $\approx 0.4^{\circ}$ in the opposite direction (minima). For particles located within the central $36 \mathrm{~cm}$ of the sphere the truncation angle is under $3^{\circ}$ in either direction with a minimum of $0.8^{\circ}$ in the sphere
A)
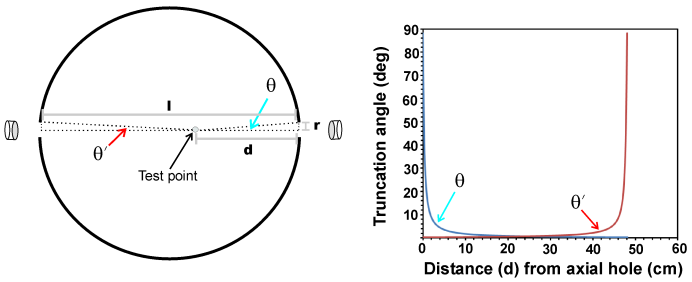

B) Homogeneous Fill Mode

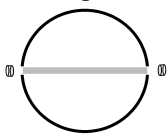

-Ignores the position of individual particles

-Assumes extinction and scattering is generated equally from all probed volume elements

\section{C) Discrete Particle Position Model}

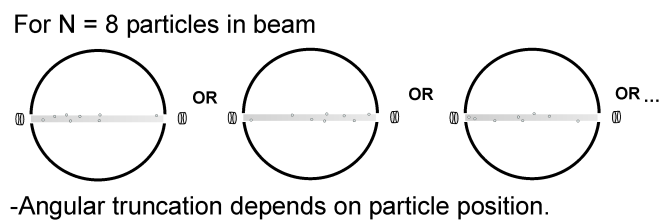

-Assumes extinction and scattering is NOT generated equally from all probed volume elements

-Model randomly distributes test particles in space

FIG. 1 (A) Left - Schematic of integrating sphere and definition of $r, d, l, \theta$ and $\theta^{\prime}$ in relation to a "test point" where a particle is presumably located. Right - Plot of "forward" $(\theta)$ and "reverse" $(\theta$ ') truncation angle vs. distance from an axial hole. (B) Illustration of the "homogeneous fill" model of aerosol scattering / extinction. The central premise is light scattering originates equally from each illuminated volume element (grey shaded area). (C) Illustration of the Discrete Particle Position Model. In this model, scattering is generated from individual points (particles) in space. Here $\mathrm{N}=8$ particles are considered, but the model can be applied for any $\mathbf{N}$. Truncation angle varies with particle position according to Eq. (2), and truncation error will also vary with particle position. Required truncation correction factors for each $\mathrm{N}=8$ case illustrated may not be equivalent.

center. The spatially averaged truncation angle for the sphere is $2.3^{\circ}$ and this is lower than the truncation angles obtained from commercial nephelometers (typically $5-15^{\circ}$ ), indicating that the spherical nephelometer design does offer a technical advantage in this regard. However, a significant fraction of scattered light may still be lost - even within this narrow angular window. Determination of this fraction is crucial for accurate scattering and albedo measurements.

\subsection{Computation of correction factors by Lorenz-Mie theory}

Two models have been developed to compute correction factors $(\mathrm{C})$ for scattering measurements. The simplest we term the homogeneous fill case, and this is illustrated in Figure 1(b). In this model, we assume scattered light is generated from each volume element probed by the laser beam equally. Due to the discrete nature of particles, it is immediately clear this model is always an approximation that may be approached only at high particle concentrations. In the second model that we call the discrete particle position model (Illustrated in Figure 1(c)), we randomly assign locations of particles within the sphere. If a small number of particles are in the probe beam, an emphasis should be placed on computing truncation errors for particles at specific locations within the sphere rather than considering spatial averages as reflected in the homoge- 
neous fill case. The significance of the discrete particle model is that the correction factor will depend on the exact position of particles within the probe laser beam. To demonstrate this, Figure 1(c) illustrates three hypothetical cases for $\mathrm{N}=8$ particles in the beam; the correction factors required for each case would not be identical.

To develop these models further we have employed LorenzMie theory, originally presented by Gustav Mie [18] in 1908, and formulated for the atmospheric aerosols by van de Hulst $[19,20]$ and others [21]. This theory allows computations of scattering properties for any spherical, homogeneous particle at any wavelength with knowledge of particle size and refractive index, and thus provides an accurate and reliable tool for predicting optical properties of aerosols. The MiePlot software written by Phillip Lavin [22] was employed in this study to compute the angular scattering intensity parameters. By assuming particles of interest are spherical and internally homogeneous, scattering intensity $\left(\mathrm{W} \mathrm{m}^{-2}\right)$ as a function of scattering angle (o) was computed by MiePlot for unit irradiance. We examined angular scattering with $0.1^{\circ}$ resolution for particles with diameter ranging from $0.05 \mu \mathrm{m}$ to $20 \mu \mathrm{m}$ for a single wavelength at $532 \mathrm{~nm}$. The refractive indices (m) explored included real values (n): 1.45, 1.50, 1.52, and 1.55, and imaginary values $(\mathrm{k}): 0,0.005,0.008$, and 0.1 . We chose these sizes and refractive indices since they span typical values encountered for atmospheric aerosols. The fraction of light not collected by the integrating sphere can be computed for any truncation angle by numerically integrating the Mie intensity parameter over the truncation angle and comparing with total integrated scattering. The light scattering was treated as exhibiting radial symmetry for scaling to 3 dimensions. Since the particles scatter light forward and backward simultaneously, the full angular range $0-180^{\circ}$ was divided into two parts: forward angles $\left(0-90^{\circ}\right)$ and backward angles $\left(90-180^{\circ}\right)$, which means a particle at any location has both forward and backward truncation angles/errors. The amount of scattered intensity loss was calculated in the forward and backward directions, respectively, and summed. This allows computation of the fraction of light not collected $(F)$ by the sphere for any particle position. Within the sphere, the CRDS beam bounces back and forth between two refractive mirrors, which is the socalled "ping-pong" model of cavity ring-down spectroscopy (CRDS). The "forward" direction becomes the "reverse" direction within a few nanoseconds time. For this model, the time averaged correction factor $(C)$ for a particle with any truncation angle can be computed as the average of an "initial" correction factor $\left(\mathrm{C}_{i}\right)$ and "subsequent" correction factor $\left(\mathrm{C}_{i+\delta t}-\right.$ e.g. when CRDS beam "reverses" direction after reflection), which can be expressed in the following equations:

$$
\begin{gathered}
C_{i}=\frac{1}{\left(1-F_{i}\right)} \\
C_{i+\delta t}=\frac{1}{\left(1-F_{i+\delta t}\right)} \\
C=\frac{C_{i}+C_{i+\delta t}}{2}
\end{gathered}
$$

The $F$ - terms in these equations correspond to the fraction of light that escapes collection by the sphere. With $C_{i}$ and
$\mathrm{C}_{i+\delta t}$ computed the time-averaged $\mathrm{C}$ values could be determined. The correction factors $(C)$ were computed for particles with diameters ranging from $0.05 \mu \mathrm{m}$ to $20.00 \mu \mathrm{m}$ for several different refractive indices for a single wavelength of $\lambda=532 \mathrm{~nm}$. However, since the particle size parameter $(\alpha)$ determines angular scattering behavior, we have used this wavelength independent variable to analyze and report the data in this manuscript. Particle size parameter $(\alpha)$ is defined as the ratio of particle circumference to wavelength of light:

$$
\alpha=\frac{2 \pi r_{p}}{\lambda}
$$

In this equation, $r_{p}$ is particle radius and $\lambda$ is wavelength of light.

\subsection{Experimental measurement of optical parameters}

A detailed description of the optical arrangement for the aerosol albedometer used in this study and the specific optical method itself is given in [1]. For brevity, we will not re-hash all the details of this experiment, but instead point out any modifications to that design. The sample flow path (inlet tubing) was modified from the original publication slightly but this would not be expected to influence optical results. In addition, the optical source employed in these experiments was the $2^{\text {nd }}$ harmonic of Nd:YAG laser (Quantel, Brio) at $\lambda=532 \mathrm{~nm}$. This laser is capable of $20 \mathrm{~mJ}$ pulses of $4 \mathrm{~ns}$ duration at $20 \mathrm{~Hz}$ repetition rate. The laser fundamental line $(1064 \mathrm{~nm})$ was filtered out through use of a filter. The CRDS employed a pair of 2.54 $\mathrm{cm}$ diameter cavity ring-down high reflective mirrors $(\mathrm{R} \approx$ 0.9997) (Los Gatos Research). Light that leaked through the exit mirror was detected with a photomultiplier tube (931B, Hammamatsu) biased at negative high voltage. The CRDS output beam was attenuated by a neutral density filter prior to measurement, preventing saturation of photomultiplier. The relative humidity (R.H.), temperature (T), and pressure (P) sensors measured directly within the sphere. These sensors were monitored by the instrument control software to examine changes in temperature and pressure inside the sphere. Typical temperature inside the sphere was $20-25^{\circ} \mathrm{C}$, and pressure was approx. $0.9 \mathrm{~atm}$. Instrument calibration was accomplished using filtered air and either $\mathrm{SF}_{6}$ or R-134a, gases with Rayleigh multipliers of 6.60 and 7.25 times that of air, respectively $[23,24]$.

\section{RESULTS AND DISCUSSION}

Figure 2 shows the Mie model correction factors (C) as a function of particle size parameter $(\alpha)$ for particles with differing refractive indices assuming the homogeneous fill case illustrated in Figure 1(b). In summary, the $C$ values increase with larger $\alpha$. This is likely because particle scattering becomes increasingly concentrated in the forward direction when the particle size increases, and therefore truncation errors increase systematically with particle size. Also plotted in Figure 2 (red squares) are experimentally measured correction factors for ammonium sulfate aerosols of varying diameter. These particles were size selected with the electrostatic classifier prior to optical analysis. Table 1 also summarizes the measured 

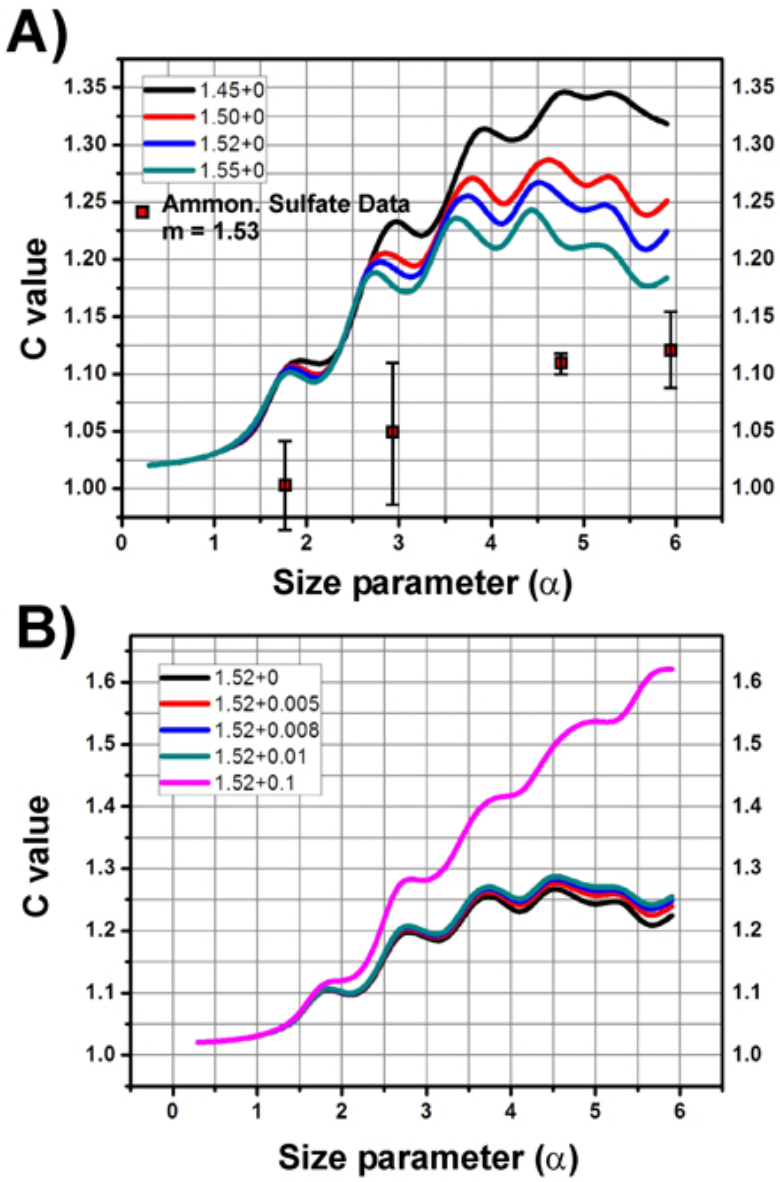

FIG. 2 (A) Plot of predicted correction factor $\mathrm{C}$ as a function of particle size parameter $(\alpha)$ for non-absorbing particles with different refractive indices. The red squares represent measured correction factors for each size with standard deviations (error bars) propagated from measurement data. (B) Plot of correction factor $\mathrm{C}$ as a function of particle size parameter $(\alpha)$ for absorbing particles with different refractive indices (the same real value but different imaginary values). The model used to generate these plots was the homogeneous fill case illustrated in Figure 1(b).

average SSA values, standard deviation and correction factors averaged over several experimental trials for different size ammonium sulfate aerosols. The data shown in the table was generated from data points reflecting values of $b_{\text {ext }}$ and $b_{\text {scat }}$ generally between $70-150 \mathrm{Mm}^{-1}$ for a particular trial. Ammonium sulfate is non-absorbing at $\lambda=532 \mathrm{~nm}$, so any measurements that reflect SSA $<1.0$ must be caused by underestimation of $b_{\text {scat }}$ due to truncation effects. As observed, small ammonium sulfate particles (diameter $=300$ $\mathrm{nm}$ ) yielded albedo values very close to 1 (essentially indistinguishable from 1). However, the measured albedo value decreased with the increase in particle size. Ammonium sulfate particles with diameter of $1000 \mathrm{~nm}$ were observed to have a mean albedo of $0.89 \pm 0.07$. The decrease in albedo (and corresponding increase in $\mathrm{C}$ ) with increasing diameter is consistent with the Mie model results. However, our light scattering model computations consistently overestimate the quantity of light lost and therefore over-predicts the required correction factor (C) compared to experimentally determined values. The discrepancy in albedo was $\approx 8 \%-11 \%$. The discrepancy between the measurements and calculation could be a result of particle longitudinal position with respect to laser beam waist

\begin{tabular}{|c|c|c|c|}
\hline Dp (nm) & $\begin{array}{c}\text { Measured SSA } \\
\text { (mean } \pm \text { std. dev. })\end{array}$ & $\mathbf{N}$ & $\begin{array}{c}\text { Measured C } \\
\text { (mean } \pm \text { std. dev. })\end{array}$ \\
\hline 300 & $0.99 \pm 0.03$ & 3 & $1.01 \pm 0.03$ \\
\hline 500 & $0.95 \pm 0.05$ & 4 & $1.05 \pm 0.06$ \\
\hline 800 & $0.90 \pm 0.004$ & 3 & $1.11 \pm 0.005$ \\
\hline 1000 & $0.89 \pm 0.03$ & 3 & $1.12 \pm 0.04$ \\
\hline
\end{tabular}

TABLE 1 Measured albedo of different size ammonium sulfate aerosols. Ammonium sulfate is non-absorbing at the measurement wavelength and theoretically should always have a single scatter albedo (SSA) of 1 . Deviation from unity indicates truncation effects. $\mathrm{N}$ - number of trials; $\mathrm{C}$ - experimentally determined correction factor; Dp particle diameter.

or perhaps non-sphericity of the salt particles. In addition, we must entertain the possibility of errors in our scattering model due to asymmetries in scattering as we scale from a 2D model calculation to 3 spatial dimensions for measurements. Nonetheless, the experimental measurements presented provide a mechanism for correction of scatter coefficient measurements made with the sphere albedometer and the magnitude of the correction scales with the Mie model results.

When the measurement chamber is filled with filtered air, instrument precision allows limits of detection $<1 \mathrm{Mm}^{-1}$ on both the extinction and scattering channels of the albedometer. When aerosol particles fill the cell, the precision can be noticeably worse (for detailed discussion see [2]). Several authors have suggested this effect results from statistical fluctuations in the number of aerosol particles within the probe beam $[2,25]$. Gaussian laser beam theory allows computation of beam diameter for a symmetric, stable resonator [26]. By applying this approach and assuming the beam is cylindrical, a beam volume of $\approx 0.02 \mathrm{~cm}^{3}$ was computed for the CRDS probe beam we employ. For particles of $800-1000 \mathrm{~nm}$ diameter, particle number densities of $80-100$ particles $/ \mathrm{cm}^{3}$ can easily produce measureable optical signals in the albedometer. Taking both the concentration and probe volume values together yields the realization that a very small number of particles (e.g. $<5$ ) are expected to occupy the probe volume at any instant. It is then clear that statistical fluctuations in particle counts in the beam at any instant may be a major source of measurement imprecision. This effect was confirmed in the previous paper published by our research group [2] in which a measurement relative standard deviation of $>0.10$ was observed for $b_{\text {scat }}$ and $b_{\text {ext }}$ when particle concentrations were low $\left(<60\right.$ particles per $\left.\mathrm{cm}^{3}\right)$ for $\mathrm{Dp}=440 \mathrm{~nm}$ ammonium sulfate.

It is clear that fluctuations in the number of particles within the beam will affect the absolute optical signals measured, what has not yet been considered is how this phenomenon will affect truncation correction factors (C). The values presented in Figure 2 represent the homogeneous fill case, but from the discussion of the previous paragraph, it is clear this is not always an appropriate model. In fact, large deviations from this model may be expected when small numbers of particles are present in the probe beam. In order to better understand the effect of number of particles within the laser beam on correction factor $(\mathrm{C})$ we have developed the discrete particle position model illustrated in Figure 1(c). Briefly, the effect 


\section{R.I. $=1.45$}
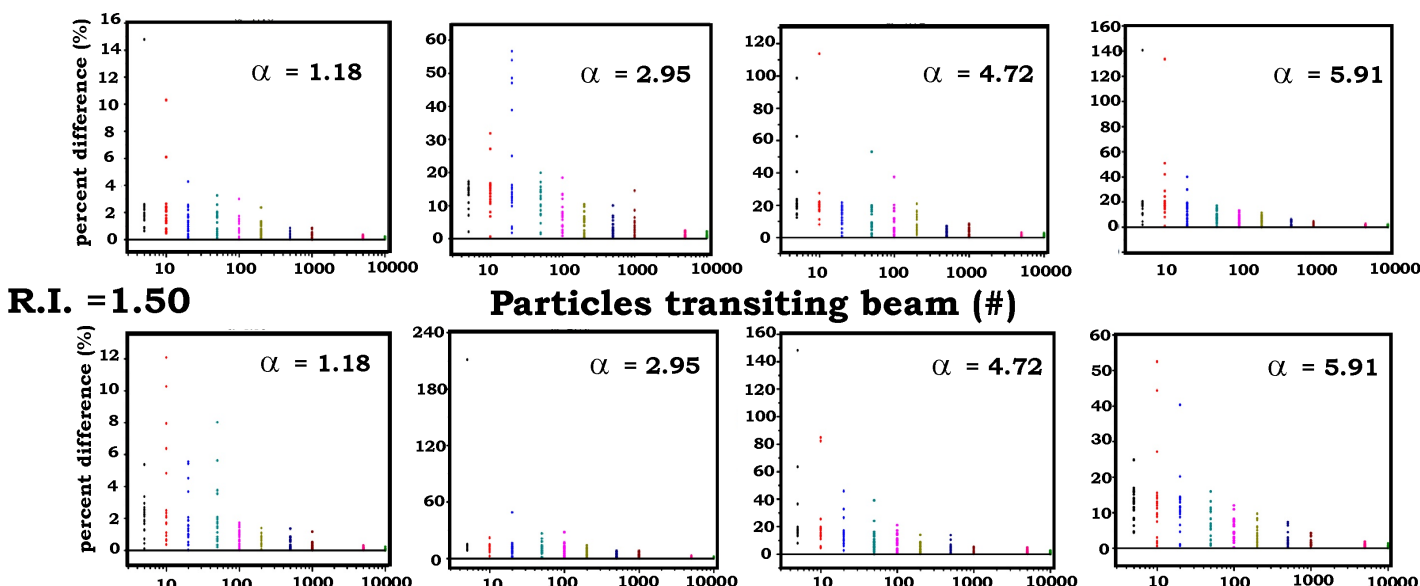

Particles transiting beam (\#)
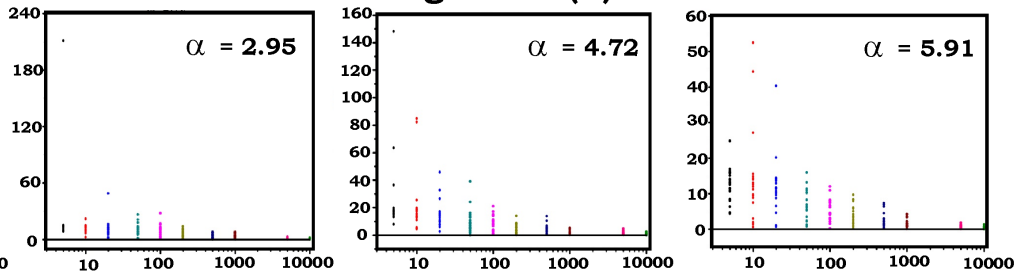

R.I. $=\mathbf{1 . 5 2}$

Particles transiting beam (\#)
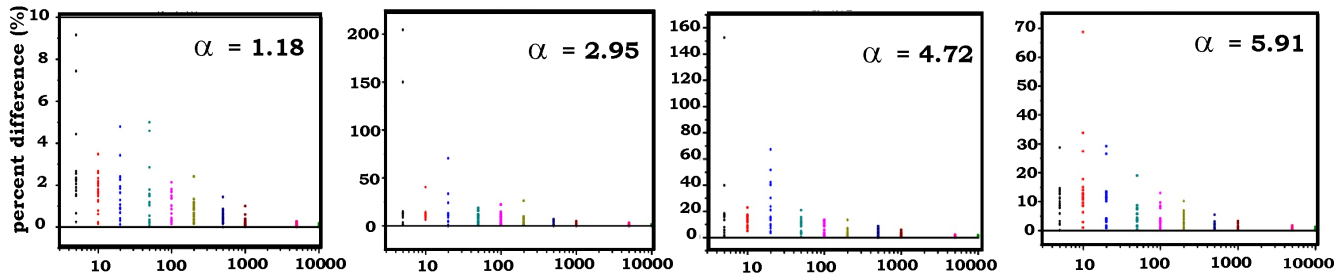

R.I. $=\mathbf{1 . 5 5}$

Particles transiting beam (\#)
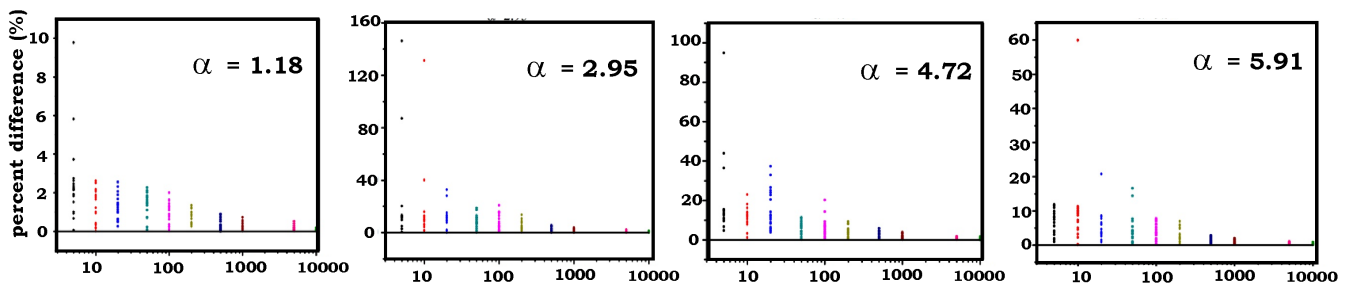

Particles transiting beam (\#)

FIG. 3 Plots of the absolute value of percent difference between discrete particle position model (Figure $1(\mathrm{c})$ ) test case with $\mathrm{N}$ particles present in the probe beam and the homogeneous fill case (Figure 1(b)) for a variety of particle refractive indices (R.I.) and size parameters $(\alpha)$. Each data point represents a single trial in which the stated number of particles were randomly arranged in the sphere. As expected, the discrete particle position model reproducibly approaches the results of the homogeneous fill case only when the number of particles transiting the beam during $\delta$ t is high. Particles transiting the beam refers to the total number of events in which a light scattering particle enters the beam.

of the number of particles within the probe beam was evaluated with respect to percent difference (\%) and relative standard deviation (RSD \%) for C values. The number of particles within the beam $(\mathrm{N})$ was varied between 5, 10, 20, 50, 100, 200, 500, 1000, 5000, and 10000 particles. These experiments were performed for spherical particles with diameter ranging from $0.05-1.00 \mu \mathrm{m}$ and with eight different refractive indices: $1.45+0 \mathrm{k}, 1.50+0 \mathrm{k}, 1.52+0 \mathrm{k}, 1.52+0.005 \mathrm{k}, 1.52+0.008 \mathrm{k}$, $1.52+0.01 \mathrm{k}, 1.52+0.1 \mathrm{k}$, and $1.55+0 \mathrm{k}$. A random number generator was used to assign random locations of $\mathrm{N}$ particles along the axial distance between the two holes located at each pole of the sphere $(\mathrm{d}=48.1 \mathrm{~cm})$. With the exact location of a particle known, truncation angle $(\theta)$, light intensity loss, and $C$ were computed via Lorenz-Mie theory. The average $C$ value was calculated for the trial considering all $\mathrm{N}$ randomly orientated particles within the probe beam (in Figure 1(c) $\mathrm{N}=8$ for example). Then, the particle positions were shuffled by re-assigning locations in the sphere by using the random number generator. The process of obtaining an average correction factor $(C)$ was then repeated. This sequence yielded 20 trials that provided 20 different average $C$ values for specific particle number, size, and refractive index. The 20 average $C$ values were compared with the $\mathrm{C}$ value obtained from the homogeneous fill case for the same particle size and refractive index condition. To compare quantitative results with the homogeneous fill case, we have chosen the variables of $\%$ difference (from homogeneous fill case) and percent relative standard deviation (\% RSD) of C. Before we begin a discussion of the results, we wish to point out that there is no fundamental difference between modeling $\mathrm{N}$ particles in the beam as a snapshot in time (as we have done), or $\mathrm{N}$ particles randomly transiting the laser beam in a time period, $\delta$ t. As such, we plot variables describing precision and accuracy in terms of "particles transiting the beam" regardless of what $\delta \mathrm{t}$ is required to achieve that condition. This will become important below when mixing of particles in the chamber is discussed.

Figure 3 illustrates plots of the absolute value of percent difference between modeled results using the discrete particle position case and the homogeneous fill case as a function of number of particles transiting the probe laser beam. The bold horizontal line at zero represents the results of the homogeneous 

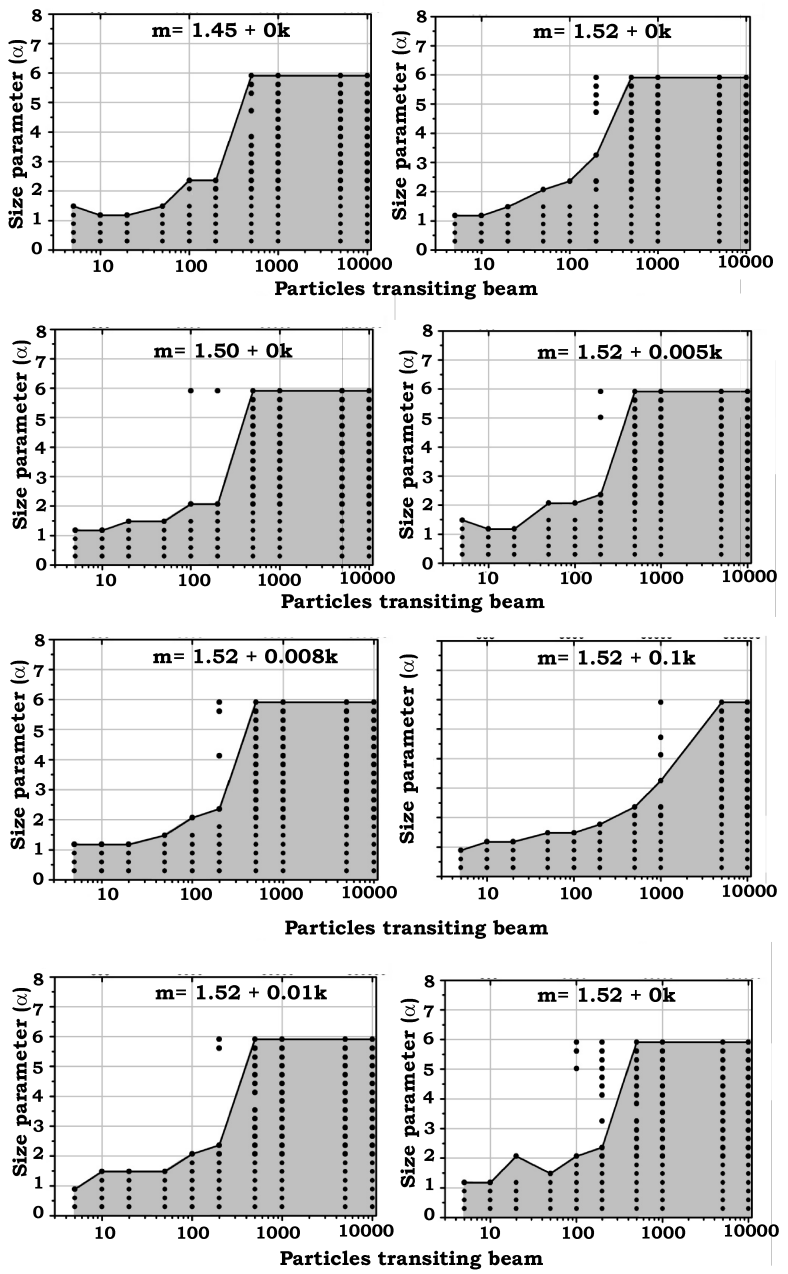

FIG. 4 Plots illustrating conditions of size parameter and number of particles transiting the probe beam that are expected to exhibit \% R.S.D. for $\mathrm{C}$ values of $\leq 5 \%$. The gray shaded areas indicate the regions where $\%$ RSD for $C$ value $\leq 5 \%$ is expected. The particles transiting the beam is a function of both particle concentration $\left(\# / \mathrm{cm}^{3}\right)$ and the measurement integration time employed to make the optical measurement.

fill case. As observed in these figures, best agreement between the two models occurs when the number of particles transiting the beam is high $(>1,000)$. If the particle transects occur randomly, then the probed volume will be adequately sampled and the homogeneous fill case will be approximated. At low numbers of particle - beam transects the predicted error between models is very high - in excess of $100 \%$ in certain cases. This indicates the homogeneous fill case would not be adequate to use for developing correction factors when the number of particle beam transects are very low. Unfortunately, the spatial randomness inherent in particle-beam-crossing events at such low numbers of transits makes developing correction factors for such cases essentially impractical. In such cases, knowledge of particle position in the sphere is required. Fortunately, actual measurement data supports the conclusion this case is not commonly encountered during measurements - even at low particle concentrations (vide infra).

The previous paragraph explored differences in the magnitude of correction factors between the homogeneous fill case and the discrete particle position case. An additional interest was to study the precision of correction factors as particles were randomly shuffled among locations within the inte- grating sphere. Insights into these results can be gained from considering the scatter of data points presented in Figure 3. At low counts of particles transiting the beam, data points appear widely scattered, indicating large variability. In an effort to be more quantitative, the relative standard deviation (\% RSD) for $\mathrm{C}$ values was determined from the 20 replicate trials for each condition of refractive index, size parameter, and number of particles transiting the beam. The large number of variables involved in this analysis (particle size, refractive index, number density etc...) makes presenting the comprehensive results of this analysis difficult in this forum. Further details of the analysis can be found in [27] which presents approx. 1600 data points and nearly 50 data plots utilized for this analysis. In addition, these modeled results are really purposed to only establish regions of particle concentration and size parameter within which reliable correction factors can be expected. With this spirit in mind, results of this analysis are summarized in Figure 4 . The gray shaded areas of this plot represent conditions of particle size parameter and number of particle beam transits $(\mathrm{N})$ where \% RSD for the $C$ value of $\leq 5 \%$ is expected. In effect, the gray shaded areas are conditions in which we expect reliable precision in correction factor to be achieved. Summarizing the trends observed for this analysis:

1. $\%$ RSD for $C$ increases with increasing particle size, but always decreases with increases in the number of particle-beam transits $(\mathrm{N})$.

2. At low N (e.g. 5, 10, 20) RSD is generally largest, indicating weak reproducibility. This is to be expected since the average truncation angle for all particles probed depends on their placement in the beam / sphere. Neither the precision of the calculated correction factor nor its accuracy is favorable at low $\mathrm{N}$.

3. For particles with any refractive index, a RSD $\leq 5 \%$ can be achieved when $\mathrm{N} \geq 5000 \mathrm{~cm}^{-3}$ and $\mathrm{RSD} \leq 2 \%$ when $\mathrm{N} \geq 10000 \mathrm{~cm}^{-3}$ can be expected. RSD $\leq 1 \%$ can be generally realized with $\mathrm{N} \geq 10,000 \mathrm{~cm}^{-3}$ particle - beam transits This is because at such large number densities, slight changes in particle position tend to average out over the large number of particles transiting the probe beam. These situations approximate the homogeneous fill case described in Figure 1(b).

4. For particles with diameter less than $0.20 \mu \mathrm{m}$ at any $\mathrm{N}$ we studied, RSD $<5 \%$ for $C$ for can be achieved. This is believed to be a result of the more isotropic directional scattering for very small particles which renders exact position of the particle in the sphere somewhat less important.

It should again be noted that this theoretical work is not a perfect quantitative model for studying the effect of number of particle transits within the probe beam on precision of $C$. However, we feel it provides a general guidance of what particle transit densities should be required to achieve precise $\mathrm{C}$ values.

To compare this model with actual experimental measurements, we have measured the relative standard deviation of 

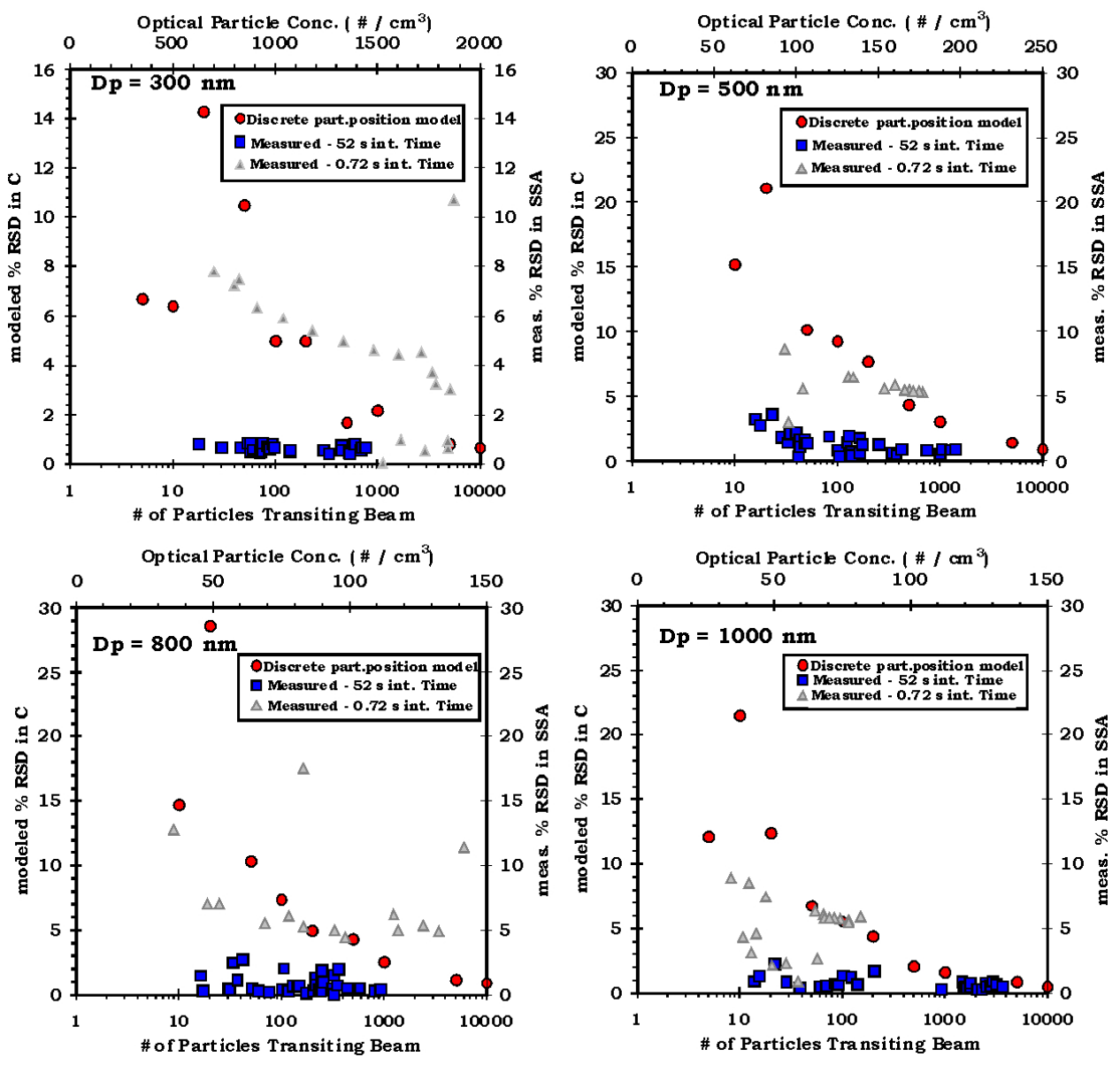

FIG. 5 Red Points - modeled \% RSD in correction factors vs. number of particles transiting the probe beam plotted on traditional $x$ and $y$ axis. This analysis is for R.I. of 1.53 (e.g. ammonium sulfate). Blue Points Second plotted on second $x$ and $y$ axis - measured \% RSD in single scatter albedo (SSA) plotted vs. the expected particle concentration derived from the optical measurement. An integration time of $52 \mathrm{sec}$ was employed. Grey points plotted on second $\mathrm{x}$ and $\mathrm{y}$ axis is measured \% RSD in single scatter albedo (SSA) plotted vs. the expected optical particle concentration with measurement integration time of only $0.72 \mathrm{sec}$. For each particle size, the $52 \mathrm{~S}$ integration time yields \% RSD data that is consistent with a very large number of particles transiting the probe beam. This suggests the homogeneous fill case is approximated at this integration time.

albedo (SSA) when size-selected ammonium sulfate (SSA = 1) aerosol filled the measurement chamber. This experiment allows us to control the diameter of the particle, use a known refractive index of the material (1.53), and even estimate the concentration of particles (particles $/ \mathrm{cm}^{3}$ ) present in the optical cell by using the measured extinction coefficient of the aerosol and the extinction cross section computed from Lorenz-Mie theory (we call this "optical particle concentration"). We can then compare measured \% relative standard deviation (\% RSD) in SSA with model results. Fluctuations in required correction factors due to particle positions in the sphere would manifest themselves as imprecision in measured SSA for a sample of constant composition. The results of this analysis are illustrated in Figure 5. The Mie model precision is reported with circles and plotted on the first $x$ and $y$ axis. The squares and triangles report measurement data for measurement integration times of 50 and $0.72 \mathrm{sec}$, respectively. The measurement data is plotted on the second $x$ and $y$ axis. Careful consideration of this figure reveals measured \% RSD values for the short integration time are much larger than for $52 \mathrm{sec}$ integration. In fact, the measured RSD values for the $52 \mathrm{~s}$ integration time are always consistent with a very large number $(>1000)$ of particle-probe beam transit events. This is found to be true for even the largest particles studied, where we expect $<5$ particles in the probe beam volume at any instant. This suggests that rapid mixing / translational motion of particles must be taking place within the sphere. The result also suggests that the $52 \mathrm{~s}$ integration time allows sufficient mixing to approximate the homogeneous fill case illustrated in Figure 1(b). In contrast, is the data presented for the $0.72 \mathrm{~s}$ measurement period. This measurement data exhibits much higher and much more variable \% RSD values for SSA. This may suggest adequate time has not elapsed to "sample" each volume element of the probe beam, and consequently the homogeneous fill case may not be appropriate at small integration times. Despite this, the measurement data for $0.72 \mathrm{~s}$ still promotes the idea of rapid mixing within the sphere. The $\%$ RSD's observed are roughly consistent with the model if roughly 5 - 15 particle beam transit events occur within the 0.72 time window even though we expect only roughly 1 particle in the probed volume at any instant based on the particle concentrations observed.

\section{CONCLUSION}

By calculating the truncation angle $(\theta)$ for our integrating sphere nephelometer, the computation of correction factors 
(C) for truncation error has been accomplished for a series of homogeneous, spherical particles with different particle sizes and refractive indices at $532 \mathrm{~nm}$ wavelength via LorenzMie theory. Modeled correction factors were generally $<1.3$ for the conditions considered. Experimental measurements of albedo for laboratory generated, size-selected ammonium sulfate aerosols $(\mathrm{m}=1.53+0.00 \mathrm{k})$ suggests correction factors of $\leq 1.12$ are required for aerosols with size parameters $<6$. Direct comparison of predicted and measured albedo values shows an average discrepancy of $8 \%-11 \%$ with the Mie model significantly over-predicting the amount of light lost from the sphere albedometer. The statistical fluctuation in the number of particles within the probe laser beam has also been considered by performing a modeling study on uncertainty in correction factor (C) caused by this effect. Results suggest $\%$ RSD $\leq 5 \%$ can be achieved when number of particle transits $(\mathrm{N})$ is $\geq 5000$, and $\% \mathrm{RSD} \leq 2 \%$ can be expected when $\mathrm{N} \geq$ 10 000. Experimental measurements of \%RSD of the measured SSA for integration times of 0.72 and $52 \mathrm{~s}$ are both consistent with rapid mixing occurring within the measurement sphere. In particular, the \%RSD values observed for the $52 \mathrm{~s}$ measurement integration time are consistent with a very large number of particle-beam transits ( $>1000)$ for all particle sizes studied. This suggests the homogeneous fill model of Figure 1(b) is appropriate at long measurement integration times, and the measured correction factors presented in Figure 2 can be considered to be good estimates to employ in correction schemes for the device.

\section{ACKNOWLEDGEMENTS}

The authors thank the State of Texas / Texas Tech University and the National Science Foundation (NSF ATM - 1004114) for financially supporting this project.

\section{References}

[1] J. E. Thompson, N. Barta, D. Policarpio, and R. DuVall, "Development of a Fixed Frequency Aerosol Albedometer," Opt. Express 16(3), 2191-2205 (2008).

[2] K. Dial, S. Hiemstra, and J. E. Thompson, "Simultaneous Measurement of Optical Scattering and Extinction on Dispersed Aerosol Samples," Anal. Chem. 82, 7885-7896 (2010).

[3] J. E. Thompson, "Aerosol Albedometer: A Tool for Measuring Optical Scattering and Extinction of Dispersed Aerosols," G.I.T. Laboratory Journal, 9-10 (2010).

[4] H. Redmond, and J. E. Thompson, "Evaluation of a Quantitative Structure Property Relationship (QSPR) for Predicting Mid-Visible Refractive Index of Secondary Organic Aerosol (SOA)," Phys. Chem. 13, 6872-6882 (2011).

[5] J. M. Haywood, and K. P. Shine, "The effect of anthropogenic sulfate and soot aerosol on the clear sky planetary radiation budget," Geophys. Res. Lett. 22(5), 603-606 (1995).

[6] J. Haywood, and 0. Boucher, "Estimates of the direct and indirect radiative forcing due to tropospheric aerosols: A review," Rev. Ceophys. 38(4), 513-543 (2000).

[7] J. D. Smith, and D. B. Atkinson, “A portable pulsed cavity ring-down transmissometer for measurement of the optical extinction of the atmospheric aerosol," Analyst 126, 1216 (2001).
[8] J. E. Thompson, B. W. Smith, and J. D. Winefordner, "Atmospheric Aerosol Measurements by Cavity Ringdown Turbidimetry," Aerosol Sci. Tech. 37(3), 221-230 (2003).

[9] T. J. A. Butler, D. Mellon, J. Kim, J. Litman, and A. J. Orr-Ewing, "Optical-feedback cavity ring-down spectroscopy measurements of extinction by aerosol particles," J. Phys. Chem. A 113(16), 3963-3972 (2009).

[10] N. Lang-Yona, Y. Rudich, E. Segre, E. Dinar, and A. Abo-Riziq, "Complex refractive indices of aerosols retrieved by continuous wave-cavity ring down aerosol spectrometer," Anal. Chem. 81(5), 1762-1769 (2009).

[11] J. E. Thompson, B. W. Smith, and J. D. Winefordner, "Monitoring Atmospheric Extinction Through Cavity Ringdown Turbidity," Anal. Chem. 74(9), 1962-1967 (2002).

[12] V. Bulatov, M. Fisher, and I. Schechter, "Aerosol analysis by cavityring-down laser spectroscopy," Anal. Chim. Acta 466, 1-9 (2002).

[13] R. Varma, H. Moosmuller, and W. P. Arnott, "Toward an ideal integrating nephelometer," Opt. Lett. 28(12), 1007-1009 (2003).

[14] J. Heintzenberg, A. Wiedensohler, T. M. Tuch, D. S. Covert, P. Sheridan, J. A. Ogren, J. Gras, et.al, "Intercomparisons and Aerosol Calibrations of 12 Commercial Integrating Nephelometers of Three Manufacturers," J. Atmos. Ocean. Tech. 23, 902-914 (2006).

[15] T. L. Anderson, and J. A. Ogren, "Determining aerosol radiative properties using the TSI 3563 integrating nephelometer," Aerosol Sci. Tech. 29, 57-69 (1998).

[16] H. E. Redmond, K. D. Dial, and J. E. Thompson, "Light scattering and absorption by wind blown dust: Theory, measurement, and recent data," Aeolian Research 2(1), 5-26 (2010).

[17] L. Ma, D. Hsieh, D. Holder, T. Zobeck, C. Morgan, and J. E. Thompson, "Optical Properties of Aeolian Dusts Common to West Texas," Aeolian Research 3, 235-242 (2011).

[18] G. Mie, "Beitrage zur Optik trüber Medien spezieller kolloidaler Metallösungen," Ann. Phys. 25, 377-445 (1908).

[19] H. C. de Hulst, Light Scattering by Small Particles (Dover, New York, 1981).

[20] R. T. Wang, and H. C. van de Hulst, "Rainbows: Mie computations and the Airy approximation," Appl. Optics 30, 106-117 (1991).

[21] C. F. Bohren, and D. R. Huffman, Absorption and Scattering of Light by Small Particles (Wiley Interscience, New York, 1983).

[22] http://www.philiplaven.com/mieplot.htm

[23] J. E. Thompson, and K. Myers, "Cavity Ring-Down Lossmeter using a Pulsed Light Emitting Diode Source and Photon Counting," Meas. Sci. Technol. 18, 147-154 (2007).

[24] S. Zadoo, and J. E. Thompson, "Rayleigh Scattering Measurements of Several Fluorocarbon Gases," J. Environ. Monitor. 13(11), 32943297 (2011).

[25] A. Pettersson, E. R. Lovejoy, C. A. Brock, S. S. Brown, and A. R. Ravishankara, "Measurement of aerosol optical extinction at $532 \mathrm{~nm}$ with pulsed cavity ring down spectroscopy," J. Aerosol. Sci. 35, 995-1011 (2004).

[26] A. E. Siegman, Lasers (University Science Books: Mill Valley, California, 1986).

[27] F. Qian, Truncation Correction for the Aerosol Albedometer: Development and Laboratory Validation of Scattering Correction Factors (M.S. Thesis, Texas Tech University, 2011). 